\title{
Química a Microscala - uma solução para um problema crítico
}

As coisas hoas surgen em pequenas quantidades (Provérbio chinês) MARGARIDA SARAIVA NEVESa. FRANCISCO J. ARNAIZb, RONALD M. PIKEG

\section{INTRODUÇĀO}

A importância dü envolver os alunos de química em actividades experimentais ć universalmente reconhccida.

Em Portugal, o reconhecimento dessa importância rellecıe-se cun vários documentos oficiais elaborados a partir las directrizes definidas pela Comissāo de Relorma do Sistema Educativo [1], no âmbito da Re[orma Educativa triciada em 1986.

Na Lei dc Bases do Sistuma Educativo (Lei $\pi^{\circ} 46 / 86$, de 14 de Outubro) encontran-se várias referências a actividades experimentais e nos programas das disciplinas das áreas de Física tr Quimica em vigor, é cufatizada a iniportancia da componente experimental scondo rornecidas orientaçō's para a realização de alguns traballıos práticos.

Contudo, é frequente encontrar alunos quc. à saída do Ensino Secundário, relertm que pouco cmvolvimeno tiveram com actividades experimentais.

São várias as razōes quc os prolessores apontam para não proporciunarem aos alunos, mais [rcquentcmente. a realização de actividades experimentais, c entre clas săo referidas os custos que as mesmas acarretain e os riscos envolvidos na sua exccusăo. Outro aspecu, de criscente importancia e que pude condicioпar stribmente a implementaçăo de actividades experimentais no futuro, é o respeitante à poluiçăo ambiental.

$O$ recurso de micruscala (utilizaçăo de pequcnas quantidades de reagentes) parece ser una resposta para alguาnas destas questöcs.

O objectivo destc artigo ć divulgar alguns dos seus asjectos, reforindo as suas potencialidades e apresentando uma bihliografia lítil para quem pretender rcalizar experiéncias de Química a micruscala.

\section{UM POUCO DE HISTÓRIA}

As primeiras releréncias a experiências de Quimica a microscala. na Europa central, datam de meados do século XIX. Pusteriormente. Emish c Pregl descnvolviram irabalhos nesta área, o que valeu a este último a atrỉbuiçāo do Prémio Nohel da Química, cm 1923.

lá no século $\mathrm{XX}$, é de realçar o Irabalho desenvolvido, neste campr, por Fcigl, e que se rellecte na sua obra iraduzida para espanhol "Analisis calitalive mediante reaciones a la yota" [2], по qual se tncontram óptimas descriçōes de materiais c métotus de trabalho a nuicroscala

Nos EUA, apris a II guerra. cm algumas instituiçōes acudémicas cnsinaram-se kécuicas de micruscala, tanto a nivel analítico como sintético. Também nas Universidades de Copenlaga e do Cairo se desenvol. veram programas nose sentido [3].

Na sequencia desses trabalhos. publicaram-se diversos artigos que pouca repercussão tiveran na altura, possivelmente derido a factores como:

- fraco reconhecimento da necessidade de prescrvar u ambiente;

- conhecimento limitato quar (4) ao perigo de manusear alguns produtos químicos:

- inexistência de balanças electrónicas monoprato, o que dificultâ. va a pesagem de prọuenas amostras.

Porcim, os movimentos ecologislas e a crescente tomada de constiencia da urgência de delender o רombience tormaram clara a necessidade de reduzir a produgão de residuns tóxicos.

Em 1982, apus muito st questionarem acerca da pertincincia da runtrodtçāo e ruadaptaçāo das túcnicas de microscala nos bahoratóríns de ensino da Química, us professures Mayo, Pike e Butcher dusenvolvcram matcriais didácticos nccessảrios à sua implementacão. A costagent destes materiais, em anos seguintes. revelou incontestáveis vantagens da microscala relativamente aos métodos tradicionais, «tudo mulito boa accitação pur parte dos alunos. Com a publicaçäo de "Microscalc Oryank laboratury" [4]. como resulnado dos irabahos daqueles professores. c: a subscquente comercialização dc equipamentos propostos nessa obra. registou-sc um crescimento expo- nencial na realizaçāo de cursos de micruscala, nes: EUA.

Em 1992, os prolessures Pike, 5zafran e Singh criaram o National Microscale Chemistry Center (NMC2) по Mcrrimack College. Notth Ardover. Massachusetts, com o objectivo de difundir o alcance e o signilicado das técnicas a microscala, organizando cursos teórico-práticos, destinados a prolessures de indos os niveis de ensino e a pessual de laboraturios industriais.

O $\mathrm{NMC}^{2}$ contribuil desde o seu іпі́cio para criaça dc uutros centros congéneres nos EUA, na Finländia. no) México na Austrâlia. na Sućcia e na Holanda e. muto recenteminte. na Índia t'em Ponto Rico.

Em 1998. a Uniẫo Europete apoinu a criação da MICRONET , Microscale Techniques Ior the Reduction of $\mathrm{Ha}$ 7arduus Wastes in Academic Laboraturies u̧ue incluti instituiçōes de tsp̧asha. Sućtia, Finlāndia. Brasil te México.

\section{O QUE É MICROSCALA?}

Nảo há concordância cunte os diversos autores quanto às quantidades de reagentes c'nvolvidas 1 um pracesso de micruscala.

Para alguns autores, numa perspectiva analíica, um เлsajo a micruscala envolve quantidadt's de amosra inlcriores a $10 \mathrm{mg}$. sendo Lrequentes consaios com golas de suluçóes. Contudo. para outros autores. do potitu de vista sintcitico, a amostra inicial poderá ir dc 25 a 250 ing para sólidos, e de 0,05 a $2 \mathrm{ml}$ para soluçōes.

A IUPAC optun pela expressão "Pechuena Escala", indicativo de sor mais importante o lacto de se registar uma relução significaıiva da quantidade de reagcontes utilizados. do que a delimileção rigorosa do campo da microscala.

\section{VANTAGENS DA QUIMICA A MICROSCALA}

Uma análise de livros americanos de experiencias de Química. pu- 
blicados nos b́ltimos ctm anos, revela não haver reduça signifjcativa das quantidades de reagentes utiliza dos, ao longo deste periodo $|5|$.

Na sala do aula. de un modu geral, o objectivo é ilustrar uma dada reacção ou a importância de uma técnica pelo qut poderá ser suficien. te a utilizaçāo do pequenas quantidades de reagentes.

Alguma rusistĉncia à adesão à microscala parcce resultar, por um lado, de st pensar que a mudança envolve muita complicaçào $e$, put outro, do desconhecimento do mutito que já se publicous sohre o assunto com uma cnorme variedade di actividades que com pouco esforço, poderāo ser adaptadas aos diversos níveis de ensinu.

A experiencia tum mostrado que a microscala é a escala óptima para a maioria das situaçōes, apresentando muito mais vantagens do que inconveniemtes, nomcadamente a nivel pedagógico.

A segurança é maior pois a exposiçăo a produtos tóxicos é diminuta, os riscos de incêndio sào redızidos e as conscquências de um tventlual acidente são mínimas. E isto tem especial impor ẫncia quando se trabalha com alunos muito jovens e com pouca experiencia em trahalho lahoratorial c 2 'm lahoratórios com infraestruturas deficientes, como infelizmente se verifica cm algumas das nossas escolas.

O tempo titilizado em montagem de equipamctomo c na execuçāo de determinadas operacōes, nas experiências a microscala, é cerca de metaue do neccessário à escala tradicional [6]. Essc ganho de tempus importante pois purmite repetir experiencias que tenham envolvido algum problema, realizar outros ensaios complementares na mesma sessão, aumentar o número de experiências a realizar. preparar t' discutir as actividades experimentais.

A microscala possibilita a imple. mentação de ensaios bascados em materiais e reagentes acessivtis a haixo custo o que origina novas possibilidades de experiencias. indo a concuntro da própria dinâni- ca do desenvolvimento científico que exige uma cada ver maior sofistica ção nas actividades [3]. Por exemplo. de um modo geral, apesar do seu intcresse actual. evita-sc propor experiências que cinvolvam metais devido au seu custo elevado; com os ensaios a microscala esse problema está ultrapassado. Além disso. individuos com alguns tipos de deficiências físicas levam a cabo mtito nats facilmente as operaçio's snvolvidas em microscala do que à escala normal

Verifica-se un maior envolvimento dos alunos. quando se thes explica as implicaçōes do recurso a microscala, possivelmenre por sentirem que estāo a agir de um modo mais racional faremdo "química verde", e por envolver haixo risco.

O trabalho a microscala contribui para uma melhor preparação dos aiunos. por um lado por promover a aquisiçùn de mator destreza no mancjo de materiais e produtos, c por outro, o ganho de termpo permite maior dıdicação à análise e discussāo de resultados, além de que coniribul para a consciencialiłaçăo da necessidade de criar novos processos químicos menos poluentes e mais adequados às necessidades da sociedadt.

Quanto às vantagens económicas é de destacar a mutnor necessidade de espaço. inferintes custos de instalaçăo e manutchçäo. " menor custo de seguros pessoais e de tedifícios e redução drástica do custo de red-

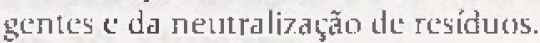

\section{A LGUNS TRABALHOS DESENVOLVIDOS NESTA ÁREA}

Un dos pioneiros mais destacados neste campo é o prolessor Thonson, da Universidado do Colorado. Em 1977. modilisou cxperjências tradicionais e aproveitol as potencialidades de materiais plásticos (tubos, seringas. ...) na concepção de experiếncias a microscala, no campo da Quimica Geral (obra 13 da Bibliografia Básica).

Existem vảrias publicasöes com óptimas descriçôes da utilização de materiais baratos para microscala, al- gumas das quais figuram na Bihliogralia Básica que mais adiante se apresenta, sendo de destacar as ohras 10., I !. e 12. Em lodas clas se podtern encontrar soluçō's engenhosas para a realização tL una grande varitodade de experiências, lais como:

- construgão e uso de capilares para a determinaçāo da Immeratura de ethuliçāo de amostras da urdem do microlitro:

- uso de capilares para derivaçāu das leis dos gases:

- construcão e uso de micropicnómetros. de microcolunas cromato gráficas, de microburctas, de equipametntos electrolíicos.

Numerosos artigos de interesse resta área aparecem regularmente jas revistas da especialidade (vejase, por exemplo. o t. Chem. Educ. on-linc). Exemplos típicos sīo as várias formas de utilizaçāo da plasticina [7], ou de vulgares halöes [8]. a construção de condensadores de ar [9] c' a preparação de instrumentos de microtiulaçāo [10].

\section{CONCLUSÃO}

Tendo em conta as vantagens apouladas para a nicroscala, é desejâvel a sua rápida adop̧çāo em todos us lahuratónios.

Essa adesảo originará a incorporação de uma grande variedade de novas experiĉncias que énriquecerá e taćlitará o ensino actualizado da Química. Lihernará majs tcmpu para rellectir sohre as expericncias e envolverá o repensar a instalaçăo e uso dos lahoratorios.

Tudo isro implicará algum esforco. mas a satisfação dos alumos na redlizaçāo das actividades e a sua melhor preparaçäo. fazem-nos sontir que a micriscale é a maneira mạis adequada para aprender quínsica te forma segura e' quase sempre estimulante.

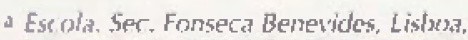

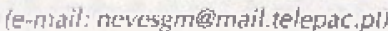

Depart. Quimica, Unis: Hurgos opool Burgos, Espanha (e-mail: ianaizoubues) Merrimak College, Natrunit Microscale Chemistry Center, Mastachuserts, EUA le-mait mpiheorpusablethomb.com
} 
BIBLIOGRAFIA BÁSICA

Até à chata foram publicados mais de 20 livros com experienciass a mícrascala, rontemplando todos os niveis de ensino da Química, e dos quais șe dẹstaca:

1. Hanwand, L. M. e Muody. C. J, 11998), Experimontal Organic chemistry: Preparative and Microncale. 2nd ed. Londres: Blackwelt Science Lid.

2. Holman. R. W. e Hesley, R. K. (1993) Contemporary Microscile Organic Chemistry, Kendall//Hunl: Dubuque, IA.

3. Mayo, D. W. Pike, R. M. e Trumper, I'. K (2000) Microscule Organic Lahoratory usth Multistep and Multiscalle Syntheses, 4 ih ed. New York. NY: Wiley.

4. Mayo, D. W., Pike, R. M. e Trumper, P. K. (2000). Microscale Technigues ror the Organic Laboratory. 2nd ed. New York, NY: Wiley.

5. Mills, I. L., Hampon, M. D. 11991), Microscale and Macroscale Expcriments for General Chemistry, 2" ed. Burf Riduc, I L: WCB/Mc Craw-itill.

6. Mohrig, J. R., Hammond, C. N. e Morrill, T. C. (1097) Ozanic Chemisiny Laboraton Manual A Balanced Aproach: Macroscte and Microscule. New York. NY: Freeman \& Co.

7. Pavia I). L. Lampman. G. M.. Kriz. G. 5 e Engell, R. G. (1999). Introduction to Organic Laboralon Techniques, A Microscale Approach. 3" ed. Philadelphia, P A: Harcourt College Publishers.

8. Singh, M. M., Pike, R. M. c Szairan, Z. (1995) Microscale and Selected Macroscdie Experiments for General and Advanced General Chemislsy. An Innowative Approach. New York, NY: Wiley.

9. Szalran, $Z_{1}$, Pike, R. M. e Singh, M. M (1991). Microscale Inorganic Chemistry: A Comprehensive Laboratory Experience. New York, NY: Wiley.

10. 5zafran, 2., Pike, R. M. e Foster, I.C (19931. Micruscale Gieneral Chemistry Laboratory with Selected Macroscale Experiments. New York, NY: Wiley.

11. Szairan. Z., Pike. R. M. e Singh, M. M. (1996). Microscale Chemistri tor High Schod. Vol. I, Kendall//Huri Pub.: Dubuque, IA.

12. Szafran, Z. Pike, R. M. e Singh, M. M. 1996). Microscale Chemistry for Migh School. Vol. Il, Kendall/ftiunt Pub.: Dubuque. IA

T3. Thompson, S (1990), Chemlrek: Small Scale Experiments for General Chemistry. NI: Prentice Hall, Upper Saddle River.
14. Williamson, K. L. \$1999/. Macroscale and Micruscale Organic Experimen/s. 3rd ed Boston, MA: Houghton Mifilin.

\section{REFERÊNCIAS BIBLIOGRÁFICAS}

i. Comissa do de Reforma do Sistema Ettucativo. Proposta Cilobal de Reforma: Relatirio rind, Lisboa, Gabinete ale Estudos e Planeamento do Miristério da Educação, 1984.

2. Feigl, F. 11949). Análisis cualitativo mediante reacciones a la gota, $3^{\text {" }}$ ed. Mad́rid: $P_{\text {a }}$ гaninfo.

3. Arnaíz, F. . e Pike R. M. 11999\}. Miencescal'a en los Laboratorios de Qumica: una revolución imparable. Anales de la Real Societlad I.s. pañola de Quimica, fulho/setembro, 45 - 51 .

4. Mayo, D. Pike. R. pe Butcher, S. (19k6). Microscde Organic Laboratory, Now York.
NY: Wiley.

5. Mayo, D.. Pike, R. E Trumper, P 12000 ) Microscale Organic Laburatory. $4^{4}$ et. New York, N.Y.: Wiley

6. Pickering. M. e LaPrade, J. E. (1986 Macro versus microlab: A controlled study of cfiticiency. J. Chem. Educ. 63. 535.

7. Arnaiz, F. J. (1998). Using plastiline in the laboratory. 1. Chem. Educ. 75 1? 1), 1418.

8. Arnaiz, F. J. (1993) Balloons in Lab. f. Chem. Educ. 70,1020 .

9. Arnaiz, F, 1. 11993) Air Condensers. f. Chem. Educ. 70, 1020 - 1021

10. Singh, M. M., McGowan, C. B. Szalran, 2. e Pike, R. M. \{1998\}. A Modilied Microburet lor Microscale titration. I. Chem. Educ. 75, 371

\section{KCNNIKK- TECH \\ Kromatografia + EspeKtroscopia}

\section{CROMATOGRAFIA: HRGC / HPLC ESPECTROSCOPIA/ESPECTROMETRIA ENGENHARIA EQUIPAMENTOS DE LABORATÓRIO PREPARAÇÃO DE AMOSTRAS CIÊNCIA DE MATERIAIS/NÁCUO}

Konik-Tech, S.A. Rua Prof. Veiga Ferreira, 6B 1600 Lisboa

Telef. 217573547

Fax. 217573485

E-mail: lishoa@kanik-uroup.com

Vendas: sales@konik-turoup.com Marketing: marketing@konik-group.com Serviço Técnico: SAT@konik-group.com www.konik-aroup.com 